LETTER

\section{A framework to a mass-dimension-one fermionic sigma model}

To cite this article: R. J. Bueno Rogerio et al2016 EPL 11360001

View the article online for updates and enhancements.
Related content

Constraining Elko dark matter at the LHC with monophoton events

Alexandre Alves, M. Dias, F. de Campos

et al.

The connection between Dirac dynamic and parity symmetry

C. H. Coronado Villalobos and R. J. Bueno

Rogerio

Exotic Elko on string-like defects in six dimensions

D. M. Dantas, Roldão da Rocha and C. A

S. Almeida

\section{Recent citations}

- Partition function for a mass dimension
$\frac{\text { one fermionic field and the dark matter }}{\text { halo of galaxies }}$
S. H. Pereira and Richard S. Costa
- Effective lagrangian for a mass dimension
$\frac{\text { one fermionic field in curved spacetime }}{\text { R.J. Bueno Rogerio et al }}$
- On the bilinear covariants associated to
$\frac{\text { mass dimension one spinors }}{\text { J. M. Hoff da Silva et al }}$

J. Hoff da Silva et al 


\title{
A framework to a mass-dimension-one fermionic sigma model
}

\author{
R. J. Bueno Rogerio ${ }^{1}$ J. M. Hoff da Silva ${ }^{1}$ S. H. Pereira ${ }^{1}$ and Roldão da Rocha ${ }^{2}$ \\ 1 Departamento de Fisica e Quimica, Universidade Estadual Paulista - Av. Dr. Ariberto Pereira da Cunha, 333, \\ Guaratinguetá, SP, Brazil \\ ${ }^{2}$ Centro de Matemática, Computação e Cognição, Universidade Federal do ABC-09210-580, Santo André, Brazil
}

received 14 January 2016; accepted in final form 24 March 2016

published online 5 April 2016

PACS 03.65.Fd - Algebraic methods

PACS 11.10.-z - Field theory

PACS $03.50 . \mathrm{Kk}$ - Other special classical field theories

\begin{abstract}
In this paper a mass-dimension-one fermionic sigma model, realized by the eigenspinors of the charge conjugation operator with dual helicity (Elko spinors), is developed. Such spinors are chosen as a specific realization of mass-dimension-one spinors, wherein the noncommutative fermionic feature is ruled by torsion. Moreover, we analyse Elko spinors as a source of matter in a background in expansion and we have found that such kind of mass-dimension-one fermions can serve not only as dark matter but they also induce an effective cosmological constant.
\end{abstract}

Copyright (C) EPLA, 2016

Introduction. - The concept of non-linear sigma models has been extensively studied in the scientific literature in a broader context. The usefulness of such framework is widely evinced from the symmetries and interconnections among different areas that it allows. Usually, apart from outstanding applications in string theory (see [1] and references therein), non-linear sigma models have served to evidence the interplay between target spaces and UtiyamaYang-Mills theories [2].

A more subtle issue concerning sigma models is the underlying framework to relativistic fermions. The firstorder Dirac equation is certainly an additional element of difficulty. The description of the physical reality based upon matter (fermionic fields) and its interactions mediated by Abelian and non-Abelian gauge theories, however, has motivated the study of possible sigma models in the broader context of spinor theory [3]. In ref. [3], by exploring the so-called bispinor geometry associated to the bispinor algebra, it was shown that for a particular class of spinors - whose density and pseudo-scalar density are non-null - the geometry of the physical observables space is given by a three-dimensional hyperbolic Robertson-Walker space.

Nearly ten years ago, the systematic study of Majorana spinors has been leading to the appreciation of massdimension-one spinor fields $[4,5]$, called Elko ${ }^{1}$. This field satisfies the Klein-Gordon, but not the Dirac equation. As

\footnotetext{
${ }^{1}$ Eigenspinors of the charge conjugation operator with dual helicity [4].
}

a crucial property, these spinors are neutral, under local gauge interactions, by means of the requirement that they are eigenspinors of the charge conjugation operator. In fact, Elko interactions, with matter and gauge fields of the standard model, are suppressed by at least one order of magnitude regarding the unification scale, providing an $a b$ initio origin of "darkness" of dark matter. In other words, interactions of Elko spinor fields are constrained to the Higgs field and gravitons, supplying a prominent direction towards physics beyond the Standard Model.

The theoretical formulation of completeness for these spinors is encoded in the (sub)groups (of the Lorentz group) which retain the underlying relativistic structure [6]. Starting from the usual concept of Dirac spinors as elements that carry the representation $(1 / 2,0) \oplus(0,1 / 2)$ of the Lorentz group, one can relate the different sectors of the representation space by means of the parity operator $P$. In the context of the full Lorentz group, $P$ is a discrete symmetry and its implementation in a given spinorial formulation culminates in the standard Dirac dynamical equation [7]. Nevertheless, the two parts of the representation space can be also related through the so-called "Pauli matrices magic" [8] without reference to any discrete symmetry. In this context, the resulting dynamics is not provided by the Dirac equation, but rather by the Klein-Gordon equation only. Working out the particularities of such spinor field in the second quantisation program, a violation of the full Lorentz symmetry appears in the spin sums. Interestingly enough, it is possible to show 
that the spin sums, and therefore the whole formulation, is invariant under $\operatorname{SIM}(2)$ group transformations [6]: precisely the subgroups of the Lorentz group obtained by removing the discrete symmetries [9]. Additionally, Elko can be experimentally produced by Higgs interactions [10,11].

In the standard model of particle physics, all regarded spinors are Dirac, Weyl or Majorana ones. Such spinors obey a first-order derivative field equation. This characteristic implies a quantum propagator that, for large momentum, is proportional to $p^{-1}$. This asymptotic behaviour of the associated propagator results, among other things, in the fact that mass dimension must be $3 / 2$. Now, the unique kinematic operator that is satisfied by Elko is the Klein-Gordon equation, a second-order derivative field equation. For this case, for a large momentum, the quantum propagator is proportional to $1 / p^{2}$, contradicting the previous case. The Klein-Gordon operator is the proper kinetic operator for Elko fields. We are, therefore, led to conclude that the mass dimension of the Elko field is one, rather than $3 / 2$, as it would be usually expected for a fermionic field.

All the above-mentioned physical aspects of these fields (mass dimension one, neutrality, spin 1/2) enable Elko spinors to be dark-matter candidates, constructed from the very first principles. Hence the systematic investigation of these dark-matter candidates, slightly and safely departing from the usual quantum description has led us to the analysis of the mass-dimension-one fermionic sigma model presented here.

In this paper we construct and investigate a non-linear sigma model associated to mass-dimension-one spinor fields. Recent formal results point to the fact that there are many kinds of such spinors [12]. However, to fix ideas, we shall report on Elko spinors, which are prototypes of mass-dimension-one fermionic fields. By sigma model we mean the mapping of the Minkowski space into a complete target space, performed by spinor fields, and its relationship with Utiyama-Yang-Mills theory. In this vein, bearing in mind the Grassmannian character of the spinor variable, we further endow the target space with torsion. We organise this paper as follows: in the next section we depict the general set up of the non-linear sigma model and its relation to non-Abelian gauge theories, also endowed to torsion in order to encompass the noncommutative fermionic aspect. Moving forward, in the third section we construct a representative sigma model for mass-dimension-one fermions. In the final section we conclude. Whenever it is possible, we provide some starting points to the application of the general formulation in cosmology.

The general setup with torsion. - This section shall present a straightforward generalisation, regarding the interplay between non-linear sigma models and non-Abelian theories [13], encoding torsion terms. As remarked in the Introduction, we envision further applications to a specific, although essentially fermionic, case.
We start by depicting the general aspects, not particularising to mass-dimension-one fermionic fields immediately. Let $\left\{\xi_{i}\right\}$ be the canonical basis of a natural inertial frame in the target space $\Sigma$, and $\left\{\mathrm{d} \xi^{i}\right\}$ its dual basis. It is possible to split the target space geometry by defining an effective metric $g \in \sec \left(T_{p} \Sigma\right)^{*} \times \sec \left(T_{p} \Sigma\right)^{*}$, where, as usual, $p$ is an arbitrary point belonging to $\Sigma$, and $\sec T_{p} \Sigma$ is a section of the tangent bundle of $\Sigma$ at $p$, such that given $\phi=\varphi^{i} \xi_{i}$, it yields

$g(\varphi, \varphi)=\left[g_{m n} \mathrm{~d} \xi^{m} \otimes \mathrm{d} \xi^{n}+\gamma_{m n} \mathrm{~d} \xi^{m} \wedge \mathrm{d} \xi^{n}\right]\left(\varphi^{i} \xi_{i}, \varphi^{j} \xi_{j}\right)$

being $\otimes$ and $\wedge$ the tensor and the exterior product, respectively. The geometrical splitting is, indeed, fulfilled by eq. (1). A direct computation of (1), taking into account the anti-symmetry relation between the two products, leads to

$$
g(\varphi, \varphi)=g_{i j} \varphi^{i} \varphi^{j}+\frac{1}{2} \tilde{\gamma}_{i j} \varphi^{i} \varphi^{j}
$$

where $\tilde{\gamma}_{i j}=\gamma_{i j}-\gamma_{j i}$. By writing a given product as its commuting and anti-commuting counterparts, i.e. $\varphi^{i} \varphi^{j}=$ $\frac{1}{2}\left[\varphi^{i}, \varphi^{j}\right]+\frac{1}{2}\left\{\varphi^{i}, \varphi^{j}\right\}$, it yields

$$
g(\varphi, \varphi)=\frac{1}{2} g_{i j}\left\{\varphi^{i}, \varphi^{j}\right\}+\frac{1}{4} \tilde{\gamma}_{i j}\left[\varphi^{i}, \varphi^{j}\right]
$$

It is worth mentioning that a thorough classification of spinors based upon bilinear covariants in a spacetime wherein the metric has both symmetric and antisymmetric parts has been accomplished in [14].

A complete sigma model, in the sense of eq. (3), can be thus studied, by means of the free Lagrangian

$\mathcal{L}=\frac{1}{2} g\left(\partial_{\mu} \varphi, \partial^{\mu} \varphi\right)=\frac{1}{4} g_{i j}\left\{\partial_{\mu} \varphi^{i}, \partial^{\mu} \varphi^{j}\right\}+\frac{1}{8} \tilde{\gamma}_{i j}\left[\partial_{\mu} \varphi^{i}, \partial^{\mu} \varphi^{j}\right]$

where Greek indexes stand for space-time coordinates. Obviously, in the usual commutative case, it yields $\left[\partial_{\mu} \varphi^{i}, \partial^{\mu} \varphi^{j}\right]=0$, hence we are simply left with $\mathcal{L}=$ $\frac{1}{2} g_{i j} \partial_{\mu} \varphi^{i} \partial^{\mu} \varphi^{j}$. In this last case, the connection with Utiyama-Yang-Mills theories is determined by the requirement $\frac{\delta \mathcal{L}}{\delta \varphi_{i}}=0$ (under space-time volume integration). In this vein, the Christoffel symbols $\Gamma_{j k}^{i}(\varphi)$ are automatically generated, in terms of which the connection $A_{\mu k}^{i}(\varphi)=\Gamma_{j k}^{i}(\varphi) \partial_{\mu} \varphi^{j}$ is identified. The equation of motion, then, reads

$$
D_{\mu j}^{i}\left(\partial^{\mu} \varphi^{j}\right)=0
$$

where the covariant derivative is given by $D_{\mu j}^{i}=\partial_{\mu} \delta_{j}^{i}+$ $A_{\mu j}^{i}$. Besides, the contraction of the Riemann curvature tensor $R_{j k l}^{i}$ with $\partial_{\mu} \varphi^{k} \partial_{\nu} \varphi^{l}$ leads to the non-Abelian field strength

$$
R_{j k l}^{i}\left(\partial_{\mu} \varphi^{k}\right)\left(\partial_{\nu} \varphi^{l}\right)=\partial_{\mu} A_{\nu j}^{i}-\partial_{\nu} A_{\mu j}^{i}+\left(\left[A_{\mu}, A_{\nu}\right]\right)^{i}{ }_{j} .
$$


Returning to the complete case, including the noncommutative sector, the functional variation of the Lagrangian leads to

$$
\partial_{\mu} \partial^{\mu} \varphi^{m}+\tilde{\Gamma}_{i j}^{m}(\varphi) \partial_{\mu} \varphi^{i} \partial^{\mu} \varphi^{j}=0
$$

with

$$
\tilde{\Gamma}_{i j}^{m}(\varphi)=\Gamma_{i j}^{m}(\varphi)+\Lambda_{i j}^{m}(\varphi)
$$

where $\Lambda_{i j}^{m}(\varphi)$ is defined as

$$
\Lambda_{i j}^{m}(\varphi) \equiv \frac{1}{2} g^{m k}\left(\partial_{i} g_{j k}-\partial_{j} g_{i k}-\frac{1}{2} \partial_{k} \tilde{\gamma}_{i j}\right)
$$

and we have the explicit contribution of the torsion terms. Moreover, the general target space curvature tensor is given by

$$
\tilde{R}_{j k l}^{i}=R_{j k l}^{i}+\partial_{[k} \Lambda_{j l]}^{i}+\left(\Gamma_{m[k}^{i}+\Lambda_{m[k}^{i}\right) \Lambda_{j l]}^{m}+\Lambda_{m[k}^{i} \Gamma_{j l]}^{m} .
$$

Notice that if $\tilde{\gamma}_{i j}=0$, and requiring $\left[\partial_{\mu} \varphi^{i}, \partial^{\mu} \varphi^{j}\right]=0$, (culminating with $\Lambda_{i j}^{m}(\varphi)=0$ ), eqs. (7) and (10) reduce to the usual case, as expected. Besides, as a matter of fact, it is not trivial (and, perhaps, not even insightful) to find the Yang-Mills counterpart of the geometric quantities as in (6), for the case at hand. The important aspect to be stressed here is that, when commutativity is lifted in the target space, torsion terms are generated.

In order to envisage the implementation of an application, we depict some cosmological implications of the sigma model. We shall briefly present the main equations that must be considered, given a specific form for the target space fields. In a curved background the action containing the corresponding contribution of the sigma model Lagrangian (4) reads

$$
S=\int \sqrt{-\bar{g}} \mathrm{~d}^{4} x\left[-\frac{R}{2 \kappa}+\frac{1}{2} h_{i j} \partial_{\mu} \varphi^{i} \partial_{\nu} \varphi^{j} \bar{g}^{\mu \nu}-W(\varphi)\right],
$$

where $R$ is the Ricci scalar, $\kappa=8 \pi G$ and $h_{i j}=g_{i j}+\frac{1}{2} \tilde{\gamma}_{i j}$ can be obtained from the symmetric and anti-symmetric property of (3). The space-time metric is represented here by $\bar{g}^{\mu \nu}$ and $W(\varphi)$ stands for a self-interacting potential.

The variation of action (11), with respect to the metric $\bar{g}^{\mu \nu}$, leads to the Einstein equations

$$
R_{\mu \nu}-\frac{1}{2} \bar{g}_{\mu \nu} R=2 \kappa T_{\mu \nu}
$$

where $R_{\mu \nu}$ is the Ricci tensor, and $T_{\mu \nu}$ is the canonical energy-momentum tensor corresponding to the matter content:

$T_{\mu \nu}=h_{i j} \partial_{\mu} \varphi^{i} \partial_{\nu} \varphi^{j}-\bar{g}_{\mu \nu}\left[\frac{1}{2} h_{i j} \partial_{\alpha} \varphi^{i} \partial_{\beta} \varphi^{j} \bar{g}^{\alpha \beta}-W(\varphi)\right]$.
Variation with respect to $\varphi^{k}$ leads to the equation of motion for the fields,

$$
\frac{1}{\sqrt{-\bar{g}}} \partial^{\mu}\left(\sqrt{-\bar{g}} \partial_{\mu} \varphi^{m}\right)+\tilde{\Gamma}_{i j}^{m}\left(\partial_{\mu} \varphi^{i} \partial^{\mu} \varphi^{j}\right)+\left(\partial_{k} W\right) g^{k m}=0
$$

with $\tilde{\Gamma}_{i j}^{m}$ given by (8).

It is straightforward to check that (14) reduces to (7), when the space-time metric $\bar{g}^{\mu \nu}$ is the Minkowski flat metric and the potential is null. By specifying the fields $\varphi^{i}$, and the potential $W(\varphi)$, we can obtain the Friedmann equations from (12). An emergent universe, supported by a non-linear sigma model without torsion, was studied in [15]. In the context approached here, by taking advantage of the fact that $h_{i j}$ encodes torsion terms, it is quite plausible that the dynamics of the evolution will be changed.

Building up the sigma model. - Part of the structure of Elko spinors, $\lambda$, is built upon the requirement $C \lambda= \pm \lambda$, being $C$ the charge conjugation operator. There exists the self-conjugated spinors $\lambda_{\alpha}^{S}\left(C \lambda_{\alpha}^{S}=+\lambda_{\alpha}^{S}\right)$ and the anti-self-conjugated spinors $\lambda_{\alpha}^{A}\left(C \lambda_{\alpha}^{A}=-\lambda_{\alpha}^{A}\right)$. Moreover, a quite judicious analysis shows that the right dual to $\lambda$ (from the relativistic point of view) reads $\vec{\lambda}_{\alpha}=$ $\pm i\left[\lambda_{\beta}\right]^{\dagger} \gamma^{0}[16]$, where the labels $\alpha$ and $\beta$ denote different types of spinors and, clearly, the dual relation stands for both self- and anti-self-conjugated spinors. As a last necessary remark, we remember that there are four different Elko spinors: two of them corresponding to different states for the self-conjugated case, and similarly to the anti-self-conjugated case [4].

It is possible to provide a particular basis adapted to eigenspinors of the charge conjugation operator, related to the Majorana representation. Alternatively, we can use the chiral representation, paradigmatically explored in all the literature of Elko. Whatever the basis is chosen, it is worth to mention that our approach is basis independent. It is useful for our purposes to construct all the possible spinors as follows: let $c^{i}$ and $d_{j}$ be $c$-numbers and write $\lambda=c^{i} \lambda_{i}$ and $\vec{\lambda}=d_{j} \vec{\lambda}^{j}$. Now let us decompose $\lambda$ (and $\vec{\lambda}$ ) in terms of the usual canonical basis (and its corresponding dual basis) as

$$
\begin{aligned}
\lambda_{i}= & \left(\begin{array}{c}
\lambda_{i}^{1} \\
\lambda_{i}^{2} \\
\lambda_{i}^{3} \\
\lambda_{i}^{4}
\end{array}\right)=\lambda_{i}^{1}\left(\begin{array}{l}
1 \\
0 \\
0 \\
0
\end{array}\right)+\cdots+\lambda_{i}^{4}\left(\begin{array}{l}
0 \\
0 \\
0 \\
1
\end{array}\right), \\
\neg^{j}= & \left(\begin{array}{llll}
\neg^{j} & \neg^{j} & \neg^{j} & \neg_{2}^{j} \\
\lambda_{1} & \lambda_{3}, \lambda_{4}
\end{array}\right)=\lambda_{1}^{j}(1,0,0,0)+\cdots \\
& +\neg_{4}^{j}(0,0,0,1) .
\end{aligned}
$$

Hence, by denoting the element basis by $\left\{\xi_{a}\right\}$ (and the corresponding dual by $\{\stackrel{\vec{\xi}}{b}\})$, we have $\lambda=c^{i} \lambda_{i}^{a} \xi_{a}$ and 
$\vec{\lambda}=d_{j} \vec{\lambda}_{b}^{j} \neg^{b}$, with $\stackrel{\neg}{\xi}^{b}\left(\xi_{a}\right)=\delta_{a}^{b}$. In order to properly implement the sigma model to the case at hand, it is necessary to modify the tensor and exterior products, encompassing the fermionic character of the fields.

We start by defining the product $\tilde{\otimes}$ in the following way: let $\mathbb{S}$ be the complex vector space generated by all the finite linear combinations of the usual Cartesian products $\left(v_{i}, \vec{v}^{i}\right)$, where $v_{i}$ and $\vec{v}^{i}$ are spanned by the respective canonical basis. Besides, take $\mathbb{I}$ as the subspace of $\mathbb{S}$ generated by

$$
\begin{aligned}
& \left(v_{i}+u_{i}, \vec{w}^{i}\right)-\left(v_{i}, \vec{v}^{i}\right)-\left(u_{i}, \vec{v}^{i}\right), \\
& \left(v_{i}, \vec{w}^{i}+\vec{u}^{i}\right)-\left(v_{i}, \vec{u}^{i}\right), \\
& \left(k v_{i}, l \vec{w}^{i}\right)-k l\left(v_{i}, \vec{w}^{i}\right),
\end{aligned}
$$

being $k, l c$-numbers and $v_{i}, u_{i}$, and so on, spanned by means of the canonical basis (similarly for the dual case). The product $\tilde{\otimes}$ is defined by conjugating elements in the space $\mathbb{S} / \mathbb{I}$, and, therefore, eqs. (17)-(19) ensure bilinearity. Hereupon, we shall pinpoint some important remarks in order to clarify the relevant properties of $\tilde{\otimes}$. Obviously, a basis of $\mathbb{S} / \mathbb{I}$ is given by $\left\{\xi_{i} \tilde{\otimes} \bar{\xi}^{i}\right\}$. We define the action of $\tilde{\otimes}$ as

$$
(v \tilde{\otimes} \vec{w})(\vec{u}, x)=\left(v^{i} \xi_{i} \tilde{\otimes} \vec{w}_{j} \vec{\jmath}^{j}\right)\left(\vec{u}_{k} \vec{̆}^{k}, x^{l} \xi_{l}\right)=v^{i} \vec{w}_{j} \vec{u}_{i} x^{j}
$$

where $\vec{w}_{j}$ and $\vec{u}_{j}$ are just coefficients and, more importantly, we have defined the action on the basis as

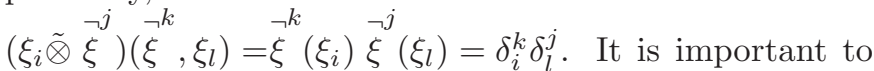
stress that the product $\tilde{\otimes}$ is unique, up to isomorphisms ${ }^{2}$. Finally, let $\left\{\xi_{i}\right\} \cup\left\{\xi_{i} \tilde{\otimes} \stackrel{\neg}{\xi}^{j}\right\}$ over the field $\mathbb{C}$ be the basis of $\tilde{\mathbb{T}}$ and $\tilde{\mathbb{I}}$ the bilateral ideal generated by $\xi_{i} \tilde{\otimes} \bar{\xi}^{i}$. The product $\tilde{\wedge}$ acting on $\tilde{\mathbb{T}} / \tilde{\mathbb{I}}$ related to $\tilde{\otimes}$ is, as expected, given by $\xi_{m} \tilde{\wedge} \stackrel{\natural}{\xi}^{n}=\frac{1}{2}\left(\xi_{m} \tilde{\otimes} \stackrel{\natural}{\xi}^{n}-\xi_{n} \tilde{\otimes} \stackrel{\xi}{\xi}^{m}\right)$.

We are now in the position to implement the splitting of eq. (1), although this time endowed with the tilde products. Hence it yields

$$
\mathcal{L}=\frac{1}{2} g_{n}^{m} \xi_{m} \tilde{\otimes} \stackrel{\neg}{\xi}^{n}\left(\partial^{\mu} \vec{\lambda}, \partial_{\mu} \lambda\right)+\frac{1}{2} \gamma_{n}^{m} \xi_{m} \tilde{\wedge} \stackrel{\neg}{\xi}^{n}\left(\partial^{\mu} \vec{\lambda}, \partial_{\mu} \lambda\right) .
$$

According to our previous construction, the fermionic decomposition along with the bilinearity of tilde products can be used in a fairly direct fashion. It is necessary, however, to call attention to the fact that the Fermi-Dirac statistics is a key feature of the Elko formulation [4], which one cannot preclude. Therefore, in the adopted decomposition $\lambda=c^{i} \lambda_{i}^{a} \xi_{a}$ and $\vec{\lambda}=d_{j} \neg_{b}^{j} \vec{\xi}^{b}$ the terms $\lambda_{i}^{a}$ and $\vec{\lambda}_{b}^{j}$ are understood as Grassmannian variables ${ }^{3}$. Taking advantage

\footnotetext{
${ }^{2}$ From a complementary point of view, the defined product $\tilde{\otimes}$ is just an isomorphism of the usual tensor product $\otimes$.

${ }^{3}$ Roughly speaking this is the attempt to reproduce quantum features of the field by absorbing the creation/annihilation operators into the expansion coefficients.
}

of these remarks, the Lagrangian (21) yields

$$
\mathcal{L}=\frac{1}{2} g_{j}^{i} d_{a} c^{b} \partial^{\mu} \stackrel{\neg}{\lambda}_{i}^{a} \partial_{\mu} \lambda_{b}^{j}+\frac{1}{4} \tilde{\gamma}_{j}^{i} d_{a} c^{b} \partial^{\mu} \stackrel{\neg}{\lambda}_{i}^{a} \partial_{\mu} \lambda_{b}^{j}
$$

It is important to stress that the formal structure of the Elko spinors is constructed taking advantage of the spinor rest frame [4]. Therefore, it is quite conceivable to add a mass term in the above Lagrangian. Therefore, it reads

$$
\begin{aligned}
\mathcal{L}= & \frac{1}{2} g_{j}^{i} d_{a} c^{b} \partial^{\mu} \vec{\neg}_{i}^{a} \partial_{\mu} \lambda_{b}^{j}+\frac{1}{4} \tilde{\gamma}_{j}^{i} d_{a} c^{b} \partial^{\mu}{\stackrel{\lambda_{i}}{a}}_{\lambda_{\mu}} \lambda_{b}^{j} \\
& +m^{2} d_{j} c^{i} \neg_{a}^{j} \lambda_{i}^{a} .
\end{aligned}
$$

It serves as the starting point for the formulation of a mass-dimension-one fermionic sigma model. Generally speaking, the target space is undertaken as a coset space of the isometry by the isotropy group. In relevant cases torsion can be added to such coset spaces [17]. The Lagrangian (23) can be adjusted to encompass these situations, $\left\{\xi_{a}\right\}$ and $\left\{\stackrel{\neg}{\xi}^{b}\right\}$ being the bases in the fiber bundle formulation, accordingly. From the Lagrangian density (23), it is interesting to note that the torsion terms do affect the spin current density. In fact, the appearance of $\tilde{\gamma}_{j}^{i}$ terms in the expression below makes this point explicit:

$$
S_{\alpha \beta}^{\mu}=-\frac{1}{2} d_{a} c^{b}\left(g_{j}^{i}+\frac{1}{2} \tilde{\gamma}_{j}^{i}\right)\left[\partial^{\mu} \neg_{i}^{a} \frac{\delta \lambda_{b}^{j}}{\delta \omega^{\alpha \beta}}+\frac{\delta \vec{\lambda}_{i}^{a}}{\delta \omega_{\alpha \beta}} \partial^{\mu} \lambda_{b}^{j}\right] .
$$

We shall finalize by considering the Elko Lagrangian (23) as the source of matter, in a curved expanding background. Some care is necessary, in order to correctly write the corresponding action in such a case. First, we define the fields $\vec{\phi}_{i}=d_{a} \vec{\lambda}_{i}^{a}, \phi^{j}=c^{b} \lambda_{b}^{j}$, and introduce the covariant derivatives, by $\nabla_{\mu} \vec{\phi}_{i}=\partial_{\mu} \vec{\phi}_{i}+\vec{\phi}_{i} \Gamma_{\mu}$, and $\nabla_{\mu} \phi^{j}=\partial_{\mu} \phi^{j}-\Gamma_{\mu} \phi^{j}$, where $\Gamma_{\mu}$ stands for the spin connections coupling Elko spinors to the background metric. The action in a curved background reads

$$
S=\int \sqrt{-\bar{g}} \mathrm{~d}^{4} x\left[-\frac{R}{2 \kappa}+\frac{1}{2} h_{j}^{i} \nabla_{\mu} \vec{\phi}_{i} \nabla_{\nu} \phi^{j} \bar{g}^{\mu \nu}-W(\vec{\phi}, \phi)\right],
$$

where $h_{j}^{i}=g_{j}^{i}+\frac{1}{2} \tilde{\gamma}_{j}^{i}$. The equations of motion for the fields follow directly, by taking the variation with respect to $\vec{\phi}_{i}$ and $\phi^{j}$. Equation (25) may serve as the starting point to apply the formulation presented here in the cosmological context. In particular, the quartic interaction appearing due to the (spin connection) torsion contribution (as in the usual fermionic case) is generated.

In order to show an explicit contribution coming from the symmetric and anti-symmetric part of the sigmamodel target space into the cosmological equations, let us take for simplicity a set of constant Elko spinor fields, such that $\partial_{\mu} \vec{\lambda}=0=\partial^{\mu} \lambda$, and the potential as the quadratic 
one in the form $W=\frac{1}{2} m^{2} \lambda \lambda$. We can choose to write $h_{j}^{i}$ in the following simple form:

$$
h_{j}^{i}=\left(\begin{array}{cccc}
g & \gamma & \gamma & \gamma \\
-\gamma & g & \gamma & \gamma \\
-\gamma & -\gamma & g & \gamma \\
-\gamma & -\gamma & -\gamma & g
\end{array}\right) \text {, }
$$

with $g$ and $\gamma$ representing the symmetric and antisymmetric components of $h_{j}^{i}$. It may sound as an oversimplification; however (as we shall see), this particularization leads to a relevant physical consequence. In a flat, homogeneous and isotropic FRW metric, $\bar{g}_{\mu \nu}=$ $\operatorname{diag}\left[1,-a(t)^{2},-a(t)^{2},-a(t)^{2}\right]$, the spin connections $\Gamma_{\mu}$ can be determined as $\Gamma_{0}=0$ and $\Gamma_{k}=-\frac{\dot{a}}{2} \gamma^{0} \gamma^{k}$, where $\gamma^{\mu}$ are the standard Dirac matrices and the dot indicates the time derivative. Notice that, even for constant spinor fields, the spin connection term couples to the metric through the term $\vec{\phi}_{i} \Gamma_{\mu} \Gamma^{\mu} \phi^{j}$, with $\Gamma_{\mu} \Gamma^{\mu}=-\frac{3}{4} \frac{\dot{a}^{2}}{a^{2}} \mathbb{I}$ and, therefore, contributes with a time-dependent term. The action (25) can be written as

$$
S=\int \sqrt{-\bar{g}} \mathrm{~d}^{4} x\left[-\frac{R}{2 \kappa}+\Lambda_{S}(t)+\Lambda_{A}(t)-\Lambda_{m}\right] .
$$

The $\Lambda_{m}$ term comes from the potential part, namely $\Lambda_{m}=\frac{1}{2} m^{2} \vec{\lambda} \lambda$, and represents a cosmological constant term, with a dependence on the mass of the Elko spinor field. The terms $\Lambda_{S}(t)$ and $\Lambda_{A}(t)$ stand for the symmetric and anti-symmetric contributions coming from the sigma model, respectively, and can be written as $\Lambda_{S}(t)=\frac{3}{8} g C\left(d_{a}, c^{b}, \vec{\lambda}_{i}^{a}, \lambda_{b}^{i}\right) H(t)^{2}$ and $\Lambda_{A}(t)=$ $\frac{3}{16} \gamma C\left(d_{a}, c^{b}, \vec{\lambda}_{i}^{a}, \lambda_{b}^{i}\right) H(t)^{2}$, where $C\left(d_{a}, c^{b}, \vec{\lambda}_{i}^{a}, \lambda_{b}^{i}\right)$ is a constant and $H(t)=\dot{a} / a$ is the Hubble parameter. They act as an effective time-varying cosmological constant, $\Lambda_{\text {eff }}(t)=\Lambda_{S}(t)+\Lambda_{A}(t)-\Lambda_{m}$. At early times of the cosmological evolution, when $\Lambda_{S}(t)+\Lambda_{A}(t)>\Lambda_{m}$, the positive contribution from $\Lambda_{\text {eff }}$ acts as an attractive gravitational field in the geometric side of the Einstein equation, leading to a decelerating universe. As the universe expands, $H(t)$ decreases. When the condition $\Lambda_{S}(t)+\Lambda_{A}(t)<\Lambda_{m}$ is reached the universe turns to be dominated by a negative $\Lambda_{\text {eff, }}$, which implies a repulsive gravitational force, driven by a constant cosmological term $\Lambda_{m}$, in perfect agreement with the $\Lambda C D M$ model. It is straightforward to see that the constant parameters could be adjusted, in order to reproduce the transition from a decelerated to an accelerated expansion of the universe. A much richer scenery concerns the study of Elko spinor fields dynamic coupled to the gravitational field [18].

Concluding remarks. - We analyzed and studied a mass-dimension-one fermionic sigma model, realized by Elko spinors. A non-commutative fermionic feature was introduced by the prominent role of torsion. The effective connection (8) that rules the Euler-Lagrange equations (7) is defined with respect to the anti-symmetric part of the metric in the target space. Thereat, Elko spinors play the role of a source of matter in an expanding background.

By the very nature of mass-dimension-one fermions, the study of the action (25) concerning the sigma model for Elko spinors can provide further insights into the darkmatter problem. However, it is also interesting to pursue questions concerning the sigma model itself. For instance, the study of the non-minimal coupling between the Riemann tensor and four Elkos, as in the standard realization of supersymmetric sigma models. Moreover, the usual $N=1(D=4)$ supersymmetric sigma-model case also imposes the necessity of a Kaehlerian target space. Hence the investigation of what type of geometric condition may arise from the extension of the present work to the supersymmetric case is also in order. We shall delve these questions in the future.

$$
* * *
$$

The authors express their gratitude to professor José ABDAlla Helayel-Neto for the privilege of his comments and appreciation of the manuscript. JMHDS acknowledges CNPq grants No. 308623/2012-6 and No. 445385/2014-6 for partial support. RDR is grateful for the CNPq grants No. 303293/2015-2 and No. 473326/2013-2, FAPESP grant No. 2015/10270-0, and for the INFN grant "Classification of Spinors", which has provided partial support. SHP acknowledges CNPq grant No. 304297/2015-1.

\section{REFERENCES}

[1] Tseytlin A. A., Int. J. Mod. Phys. A, 4 (1989) 1257.

[2] Gell-Mann M. and Levy M., Nuovo Cimento, 16 (1960) 705.

[3] Crawford J. P., J. Math. Phys., 31 (1990) 1991.

[4] Ahluwalia D. V. and Grumiller D., JCAP, 07 (2005) 012.

[5] Ahluwalia D. V., Lee C. Y. and Schritt D., Phys. Rev. D, 83 (2011) 065017.

[6] Ahluwalia D. V. and Horvath S. P., JHEP, 11 (2010) 078.

[7] SperançA L. D., Int. J. Mod. Phys. D, 23 (2014) 1444003.

[8] Ramond P., Field Theory: A Modern Primer, second edition (Westview Press) 2001.

[9] Cohen A. G. and Glashow S. L., Phys. Rev. Lett., 97 (2006) 021601.

[10] Dias M., de Campos F. and Hoff da Silva J. M., Phys. Lett. B, 706 (2012) 352.

[11] Agarwal B., Jain P., Mitra S., Nayak A. C. and Verma R. K., Phys. Rev. D, 92 (2015) 075027.

[12] Coronado Villalobos C. H., Hoff da Silva J. M. and DA RochA R., Eur. Phys. J. C, 75 (2015) 266.

[13] Mukni S., Classical and Quantum Theory of Supersymmetric $\sigma$-Models, in Superstrings, Unified Theories 
and Cosmology, Proceedings of the Summer Workshop in High Energy Physics and Cosmology, Trieste, Italy, 30 June-15 August 1986, edited by Furlan G., Jengo R., Pati J. C., Sciama D. W., Sezgin E. and Shafi Q., The ICTP Series in Theoretical Physics, Vol. 3 (World Scientific Pub. Co., Teaneck, NJ) 1987, ISBN 9971-50-271-2, pp. $53-70$.

[14] AbŁamowicz R., Gonçalves I. and Da Rocha R., J. Math. Phys., 55 (2014) 103501.
[15] Beeshan A., Chervon S. V. and Maharaj S. D., Class. Quantum Grav., 26 (2009) 075017.

[16] Ahluwalia D. V., LeE C. Y. and Schritt D., Phys. Lett. B, 687 (2010) 248.

[17] Batakis N. A., Farakos K., Kapetanakis D., Koutsoumbas G. and Zoupanos G., Phys. Lett. B, 220 (1989) 513.

[18] Böhmer C. G., Burnett J., Mota D. F. and Shaw D. J., JHEP, 07 (2010) 053. 